\title{
Modelling of seasonal influenza and estimation of the burden in Tunisia
}

S. Chlif ${ }^{1+}$, W. Aissi ${ }^{1,2+}$, J. Bettaieb ${ }^{1,2}$, G. Kharroubi ${ }^{1,2}$, M. Nouira ${ }^{1,2}$, R. Yazidi', A. ElMoussi ${ }^{3}$, L. Maazaoui ${ }^{4}$ A. Slim ${ }^{3}$, A. Ben Salah ${ }^{1,2}$ and the Influenza Surveillance Group in Tunisia

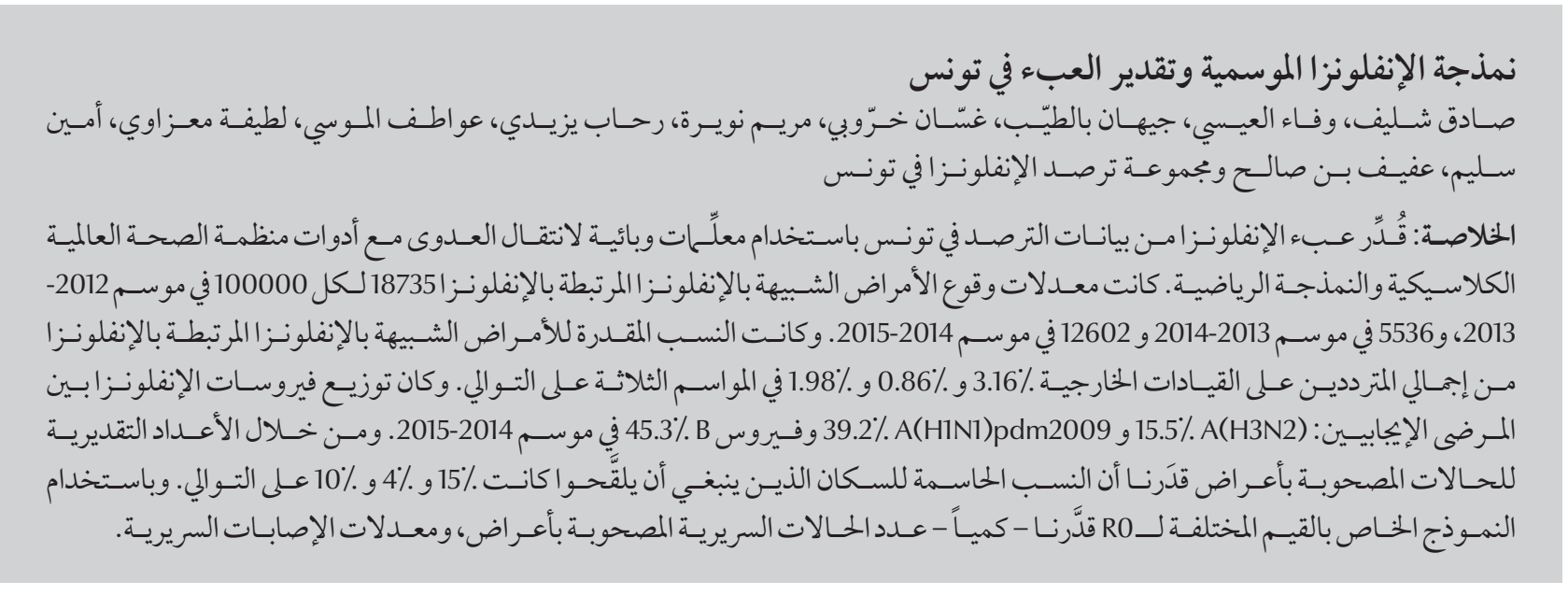

ABSTRACT The burden of influenza was estimated from surveillance data in Tunisia using epidemiological parameters of transmission with WHO classical tools and mathematical modelling. The incidence rates of influenza-associated influenza-like illness (ILI) per 100000 were 18735 in 2012/2013 season; 5536 in 2013/14 and 12602 in 2014/15. The estimated proportions of influenza-associated ILI in the total outpatient load were 3.16\%; $0.86 \%$ and $1.98 \%$ in the 3 seasons respectively. Distribution of influenza viruses among positive patients was: A(H3N2) 15.5\%; A(H1N1)pdm2009 39.2\%; and B virus 45.3\% in 2014/2015 season. From the estimated numbers of symptomatic cases, we estimated that the critical proportions of the population that should be vaccinated were $15 \%, 4 \%$ and $10 \%$ respectively. Running the model for the different values of R0, we quantified the number of symptomatic clinical cases, the clinical attack rates, the symptomatic clinical attack rates and the number of deaths. More realistic versions of this model and improved estimates of parameters from surveillance data will strengthen the estimation of the burden of influenza.

\section{Modélisation de la grippe saisonnière et estimation de sa charge en Tunisie}

RÉSUMÉEn Tunisie, la charge de la grippe a été estimée à partir des données de surveillance, en utilisant les paramètres épidémiologiques de la transmission avec les outils classiques de I'OMS et la modélisation mathématique. Les taux d'incidence des syndromes de type grippal (STG) associés à la grippe étaient 18735 pour 100000 pour la saison 2012-2013; 5536 pour 2013-2014 et 12602 pour 2014-2015. La part estimée de STG associés à la grippe pour la charge totale de patients externes était respectivement de 3,16\%, 0,86\% et 1,98\% pour les trois saisons. Parmi les patients positifs au virus de la grippe, la répartition était la suivante pour la saison 2014-2015: 15,5\% pour le virus $\mathrm{A}(\mathrm{H} 3 \mathrm{~N} 2) ; 39,2 \%$ pour le virus A(H1N1)pdm2009; et 45,3\% pour le virus B. À partir du nombre estimé de cas symptomatiques, nous avons calculé que la proportion critique de la population devant être vaccinée était respectivement de $15 \%, 4 \%$ et $10 \%$. L'exécution du modèle avec les différentes valeurs de R0 nous a permis de déterminer le nombre de cas cliniques symptomatiques, les taux d'attaque clinique, les taux d'attaque clinique pour les cas symptomatiques et le nombre de décès. Des versions plus réalistes de ce modèle ainsi que des estimations améliorées des paramètres issus des données de surveillance permettront d'accroître l'utilité des modèles mathématiques.

'Laboratory of Medical Epidemiology, Pasteur Institute of Tunis, Tunis, Tunisia (Correspondence to A. Ben Salah: afif.bensalah@pasteur.tn). ${ }^{2}$ Faculty of Medicine of Tunis, University of Tunis El Manar, Tunis, Tunisia. ${ }^{3}$ Virology Unit, Microbiology Laboratory, National Influenza Centre, Charles Nicolle's Hospital, Tunis, Tunisia. ${ }^{4}$ Primary Health Care Directorate, Minisstry of Public Health of Tunisia, Tunis, Tunisia. Additional authors listed on page $466 .{ }^{\dagger} J$ oint author

Received: 18/05/2016; accepted: 25/08/2016 


\section{Introduction}

A national programme for surveillance and control of influenza was established in Tunisia in 1980 within the Department of Primary Health Care at the Ministry of Health. It involves the National Influenza Centre, recognized by the World Health Organization (WHO) in 1980 as well as a network of 268 centres for reporting influenza-like illness (ILI), which is geographically, socioeconomically and demographically representative of the Tunisian population (1). Aggregated data on ILI cases is reported regularly from these centres in a standardized form specifying the number of ILI cases and the total number of outpatients by age $(0-5$ years; $6-16$ years and $\geq 16$ years). The National Influenza Centre receives biological specimen from the ILI centres as well as from university hospitals (mainly intensive care, pneumology or infectious disease wards) to adapt treatment for severe cases.

The analysis of collected data is performed towards the end of the season (analysis of trends, composition of serotypes, severity, geographic and demographic distribution). It is detailed per week for the ILI proportion compared with all outpatient consultations for the 24 governorates of Tunisia. However, despite its importance this information might underestimate the burden of influenza because of difficulties related to valid numerators (total ILI cases and influenza-related cases) and denominators (total exposed populations).

The aim of this study was to estimate appropriate epidemiological parameters for influenza transmission with classical epidemiological tools and mathematical modelling in order to evaluate the burden of disease using ILI sentinel surveillance data and the proportion of laboratory-confirmed influenza cases. Specifically, we evaluated the burden of seasonal influenza between 2012 and 2015, first using tools developed by $\mathrm{WHO}$ and then with mathematical modelling. The findings might be used to guide control strategies, such as the use of influenza vaccines, by testing their expected effectiveness in the reduction of influenza burden.

\section{Methods}

\section{Case definitions}

The case definition of ILI is that recommended by $\mathrm{WHO}$ : acute respiratory illness, and measured fever $\geq 38^{\circ} \mathrm{C}$, and cough, and onset in previous 10 days (2). This case definition has been used in the Tunisian surveillance system since 2014. Previously, ILI was defined as an outpatient with fever $\left(\geq 38^{\circ} \mathrm{C}\right)$ and cough or sore throat with onset less than 5 days prior to presentation in the absence of a specific diagnosis (1).

\section{Study population and area}

The study area was the districts comprising the catchment area of the 268 ILI sites for influenza surveillance in Tunisia at the community level. A description of the distribution of these centres has been detailed elsewhere (1).

\section{Estimation of disease burden}

Disease burden associated with seasonal influenza was estimated for 3 seasons (2012-2013, 2013-2014, 2014-2015) from ILI sentinel surveillance data and using tools developed by $\mathrm{WHO}(3)$

The national incidence of mild influenza was estimated from data collected (number of ILI cases) at the 268 ILI sentinel sites (1) and using the proportion of laboratory-confirmed influenza cases for each season as: influenza-associated ILI incidence rate $=$ total No. of influenza-associated ILI cases/population covered by sentinel sites.

The total number of influenzaassociated ILI cases was obtained by multiplying the number of ILI cases by the proportion of laboratory-confirmed influenza cases in each season; positivity rate (obtained by dividing the number of influenza-positive cases by the total number tested in each season according to the results of all the tests received by the National Influenza Centre from ILI sites as well as from university hospitals). We used the positivity rate of all specimens received by the National Influenza Centre and tested for influenza because of the lack of accurate information on the positivity rate specifically in the sentinel sites.

The population covered by ILI sites was provided by the Department of Primary Health Care. The age-specific incidence rate for influenza-associated ILI was estimated by the same method. The total number of influenza-associated ILI cases was calculated by age; and the agespecific population covered by sentinel sites was obtained by applying the age distribution of the Tunisian population according to the 2014 census of the population to the population covered by ILI sentinel sites. The age-specific incidence rate for influenza-associated ILI was then estimated for 2 age groups ( $0-5$ years and $\geq 6$ years) that were available in both ILI data and the published results of the 2014 census (4).

The proportional contribution of influenza-associated ILI to all outpatients was estimated by dividing the same numerator (described above) by the total number of outpatients at ILI sentinel sites for each season as follows:

\footnotetext{
Proportional contribution of influenza

-associated ILI to outpatient load (\%) Total number of influenza-associated ILI cases

Total number of outpatients visits at ILI sentinel sites
}

The proportion of influenza-associated ILI compared with total outpatient load was estimated for the age groups used in the ILI sentinel surveillance system ( $0-5$ years, $6-16$ years, $\geq 16$ years).

Estimates of the incidence and proportion of influenza-associated ILI among all cases are based on number of cases identified at sentinel sites, which is by definition a sample of the population of the catchment area. Therefore, we 
calculated the confidence interval (CI) for the estimates to account for random sampling variation. The lower bound is the rate (or proportion) divided by the error factor, and the upper bound is the rate (or proportion) multiplied by the error factor (EF) which is calculated from the exponent of 1.96 divided by the square root of $d$, the number of cases of influenza-associated ILI: EF = $\mathrm{e}(1.96 / \sqrt{d})(3)$.

The chi-squared test and corresponding P-values were used to compare the epidemiological indicators over 3 seasons.

We estimated the seasonal pattern of laboratory-confirmed influenza in the seasons 2012-2013; 2013-2014 and 2014-2015 using all samples tested for influenza by the National Influenza Centre during all the three seasons.

\section{Mathematical modelling}

A basic mathematical model that captures the natural history of influenza was developed (5), in which S refers to susceptible cases, $\mathrm{L}$ is latent (not yet infectious), I is infected (infectious), A is asymptomatic (partially infectious) and $\mathrm{R}$ is recovered or removed. The model is a general SLIAR (susceptible latent infected asymptomatic recovered or removed cases) epidemic model as proposed by Brauer (6), with the assumption that the asymptomatic fraction evolving from latent never becomes symptomatic and then moves to the removed (recovered) pool. This was used to derive the basic reproduction number, R0, and additional deaths attributable to severe disease.

The flowchart of the structure of the model is illustrated in Figure 1. Model parameter values, collected mostly from literature, are:

- Probability of developing symptoms $(p)=2 / 3(5)$

- Reduction of infectiveness for asymptomatic infections $(\delta)=0.5(5)$

- Fraction recovering from infective state $(f)=0.999$. This parameter was adjusted from 0.98 (5) to have a coherent profile with the Tunisian epidemiological situation.

- Rate of leaving the infective state $(\alpha)$ $=1 / 4.1=0.244(7)$

- Rate of leaving the asymptomatic $\operatorname{compartment}(\eta)=1 / 4.1=0.244(7)$

- Rate of leaving the latent compartment $(\kappa)=1 / 1.9=0.526(7)$

- The contact rate $\beta$ is derived from R0 using Equation 7. The initial population was taken as 11 million, which is the current size of the Tunisian population (4).

- Equations 1-6 below translate this flowchart into a set of ordinary differential equations:

with initial conditions $S(0)=S_{0}$, $\mathrm{L}(0)=0, \mathrm{I}(0)=\mathrm{I} 0, \mathrm{~A}(0)=0, \mathrm{R}(0)=0$

$$
\begin{aligned}
& \frac{d S}{d t}=-\beta S(I+\delta A)(7) \\
& \frac{d L}{d t}=-\beta S(I+\delta A) \mathrm{KL}(2) \\
& \frac{d l}{d t}=p \kappa L-\alpha /(3) \\
& \frac{d A}{d t}=(1-p) \kappa L-\eta n A(4) \\
& \frac{d R}{d t}=f \mathrm{al}+\mathrm{n} A(5) \\
& \frac{d N}{d t}=-(1-f) \mathrm{al} /(6)
\end{aligned}
$$

and $\mathrm{N}(0)=\mathrm{S}_{0}+\mathrm{I}_{0}$.

Initially, the total population size is $\mathrm{N}$, of which a small number, $\mathrm{I}$, are infective and the remainder, $\mathrm{S} 0$, are susceptible.

Using the approach described by van den Driessche (8), the model permits the calculation of the basic reproductive number, R_ 0 , defined as the number of secondary infections caused by introduction of a single infective person into a fully susceptible population. R_ 0 is expressed as follows (Equation $7 \overline{-}$

An intuitive interpretation of this basic reproduction number is that a latent

$$
R_{0}=S_{0} \beta\left[\frac{p}{a}+\frac{\delta(7-p)}{\eta}\right] \text { (7) } \quad=p\left[1-\frac{S_{\infty}}{N(0)}\right] \text { (14) }
$$

case introduced into a population of $\mathrm{S} 0$ susceptible people becomes infective with probability $\mathrm{p}$, and causes $\beta \mathrm{SO} / \mathrm{a}$ infections during the infective period of length $1 / \alpha$, or becomes asymptomatic with probability $1-p$ and causes $\delta \beta$ S0/a infections during asymptomatic period of length $\eta$ (Equation 5) (5).

Despite the nonlinearity of the system and absence of an analytical solution, the model was analysed at infinity $(t \rightarrow \infty)$. Arino et al. showed that the final size of $S$ remains strictly positive (i.e. $\left.S \_\infty>0\right)$, meaning that some members of the population remain uninfected during the epidemic $(9,10)$. Furthermore, the final size of $S$ is given by:

By neglecting the term, $\frac{\beta l_{0}}{\alpha}$ Equation 8 gives us the following estimation of the basic reproduction number:

$$
\ln \left(S_{0}\right)-\ln \left(S_{\infty}\right)=R_{0}\left[1-\frac{S_{\infty}}{S_{0}}\right]+\frac{\beta I_{0}}{a}(8)
$$

Basic morbidity and mortality indicators can also be derived from the model as shown in Equations 10-14.

$$
R_{0} \approx \frac{\ln \left(\mathbf{S}^{\circ}\right)-\ln \left(S^{\infty}\right)}{1-\frac{S_{\infty}}{S_{0}}}
$$

- No. of clinical cases during the epidemic $=\mathrm{I}_{0}+\left(\mathrm{S}_{0}-\mathrm{S}_{\infty}\right)(10)$

- No. of symptomatic cases during the epidemic $=\mathrm{I} \_0+\mathrm{p}\left(\mathrm{S}_{0}-\mathrm{S}_{\infty}\right)(11)$

- No. of deaths due to the disease during the epidemic $=(1-\mathrm{f}) \quad\left[\mathrm{I}_{0}+\mathrm{p}\left(\mathrm{S}_{0}-\right.\right.$ $\left.\left.\mathrm{S}_{\infty}\right)\right](12)$

- $\quad$ Clinical attack rate $=1-\mathrm{S}_{\infty} /(\mathrm{N}(0))$ (13)

- Symptomatic attack rate =

The symptomatic attack rate, the

$$
1-\frac{S_{\infty}}{N(0)}(13)
$$

fraction of the population that develop 


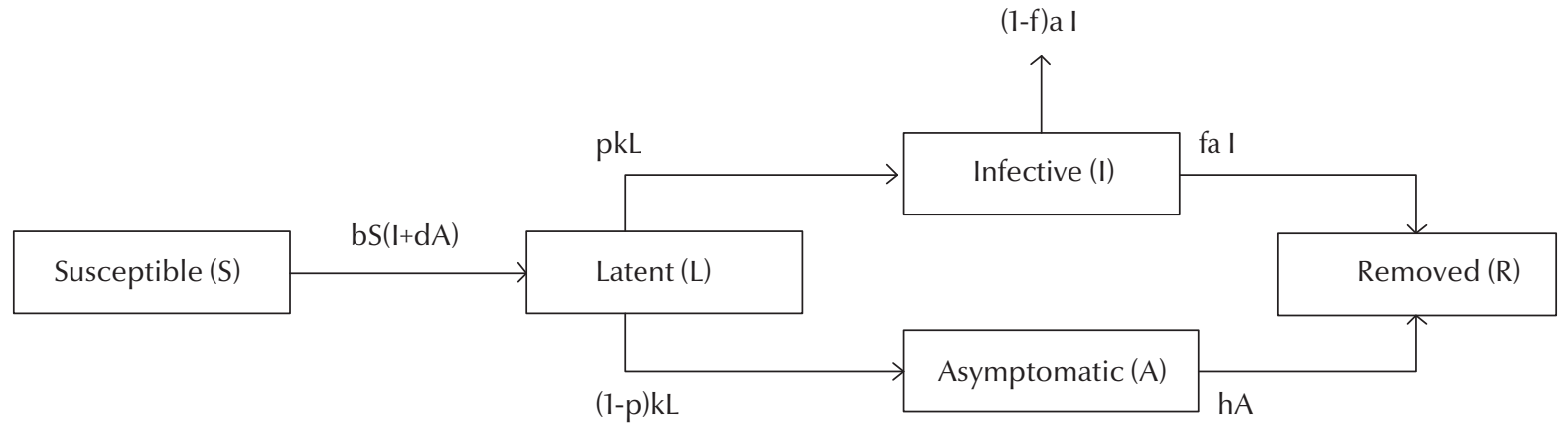

Figure 1 Influenza model flow chart

symptomatic infection, is estimated from ILI data for the 3 seasons. Using Equation 14, $S_{\infty}$ is derived from the symptomatic attack rate:

$$
S_{\infty}=N(0)\left[1-\frac{S_{\infty}}{p}\right](15)
$$

$\mathrm{R} 0$ is generated using Equation 9.

\section{Simulations of the model}

The model was solved using Berkely Madonna, version 8.3.23. A simulation was done for each of the 3 seasons. The mortality and morbidity indicators were computed using Equations 10-14.

\section{Critical vaccination coverage}

The proportion of the population to be vaccinated in order to avoid an influenza outbreak is known as the critical vaccination coverage, and is commonly denoted as pc. In other words, a minimum fraction of the population has to be moved from a susceptible state to a recovered state through vaccination in order to avoid transmission. To do so, the effective reproduction number, $\mathrm{R}_{\text {effective' }}$ has to be less than 1. Given that $\mathrm{R}_{\text {effective }}=\mathrm{R} 0 \times(\mathrm{S} / \mathrm{N})$, this can be ex-

$$
P_{c}=1-\frac{1}{R_{0}}(17)
$$

pressed as:

$$
\frac{S}{N}<\frac{1}{R_{0}}(16)
$$

This can only be reached if at least: of the population, supposedly fully susceptible, is vaccinated.

\section{Results}

During the most recent season (October 2014-April 2015), the surveillance system registered 158085 cases of ILI among 2165964 outpatients in all sentinel sites in Tunisia. In the 2012-2013 season, 170623 cases of ILI were recorded among 2023942 outpatients; and in the 2013-2014 season, there were 156513 cases of ILI among 2196 715 outpatients.

The estimated incidence rates for influenza-associated ILI (per 100 $000)$ by age group in influenza seasons 2012-2015 are shown in Table 1. The incidence rates differed significantly between the 3 seasons $(\mathrm{P}<0.0001)$, with the highest national rate in 2012-2013 and the lowest in 2013-2014. The incidence was greater among children aged $\leq 5$ years compared with that for persons aged $\geq 6$ years in all 3 seasons $(\mathrm{P}<0.0001)$.

The estimated proportions of influenza-associated ILI in the total outpatient load by age group are shown in Table 2. These proportions also differed significantly according to influenza season $(P<0.0001)$, with the highest rate in 2012-2013 and the lowest in 2013-2014. The estimated proportion of influenza-associated ILI in the total outpatient load was higher among children aged 6-16 years in all 3 seasons

Table 1 Estimated incidence (per 100 000) and number of new cases of influenza-associated influenza-like illness distributed by age group in three influenza seasons, Tunisia

\begin{tabular}{lcccccc} 
Age (years) & \multicolumn{3}{c}{ Incidence (95\% Cl) } & \multicolumn{3}{c}{ No. of new cases (95\% Cl) } \\
& $\mathbf{2 0 1 2 / 1 3}$ & $\mathbf{2 0 1 3 / 1 4}$ & $\mathbf{2 0 1 4 / 1 5}$ & $\mathbf{2 0 1 2 / 1 3}$ & $\mathbf{2 0 1 3 / 1 4}$ & $\mathbf{2 0 1 4 / \mathbf { 1 5 }}$ \\
& $P<0.0001$ & $P<0.0001$ & $P<0.0001$ & $P<0.0001$ & $P<0.0001$ & $P<0.0001$ \\
$0-5$ & 31896 & 10789 & 23278 & 310090 & 104887 & 226304 \\
& $(31265-32540)$ & $(10424-11166)$ & $(22740-23828)$ & $(303958-316347)$ & $(101346-108552)$ & $(221074-231657)$ \\
$\geq 6$ & 17457 & 5025 & 11565 & 1747567 & 503044 & 1157812 \\
& $(17310-17604)$ & $(4946-5104)$ & $(11446-11686)$ & $(1732925-1762333)$ & $(495217-510996)$ & $(1145905-1169842)$ \\
Total & 18735 & 5536 & 12602 & 2057657 & 608009 & 1384115 \\
& $(18590-18881)$ & $(5457-5615)$ & $(12484-12722)$ & $(2041764-2073675)$ & $(599398-616745)$ & $(1371091-1397264)$ \\
\hline
\end{tabular}




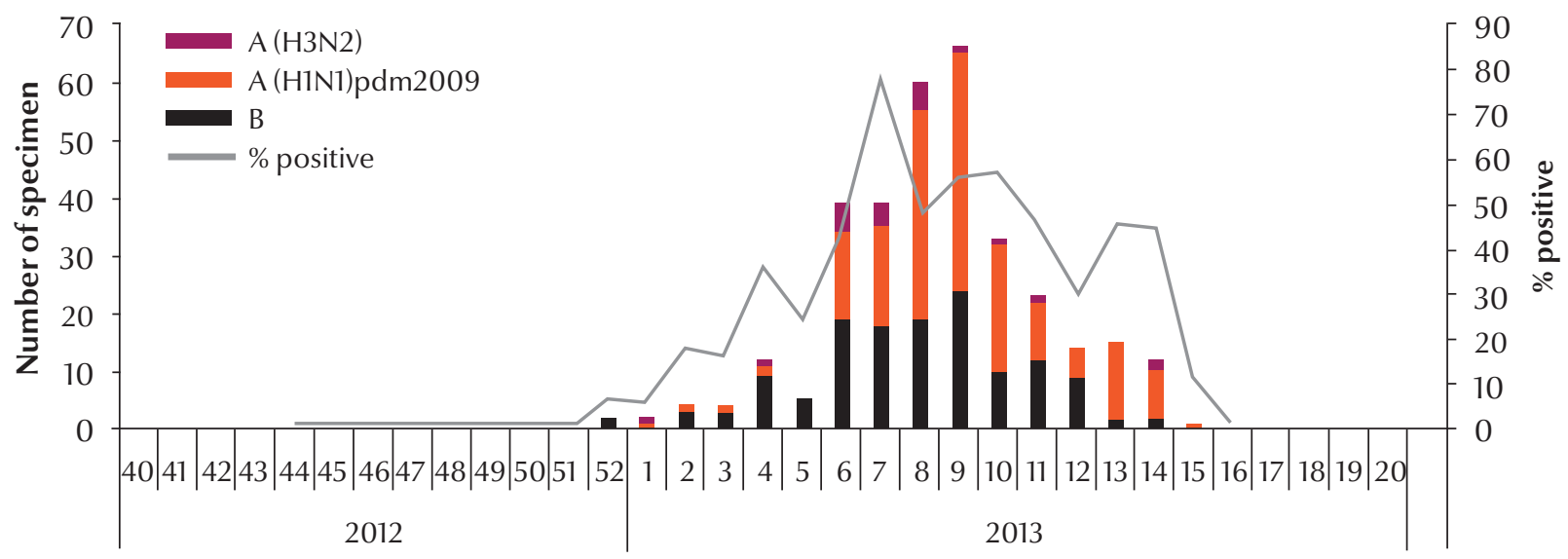

Week and year

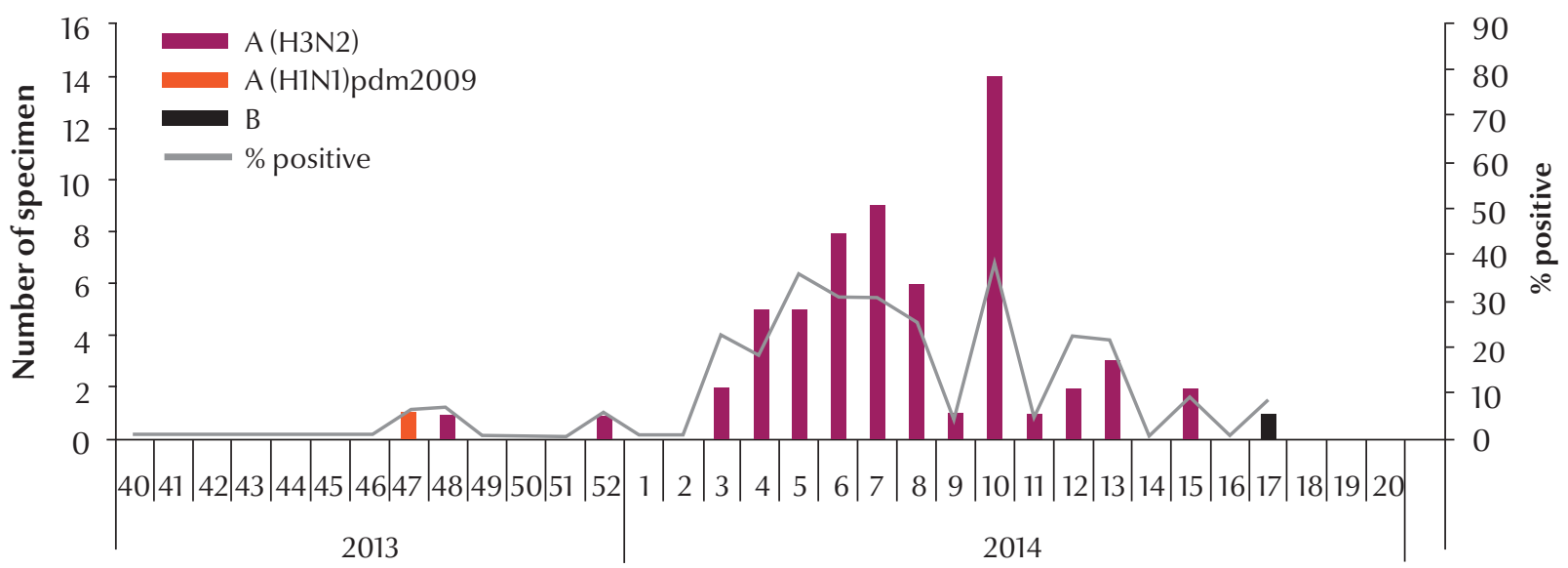

Week and year

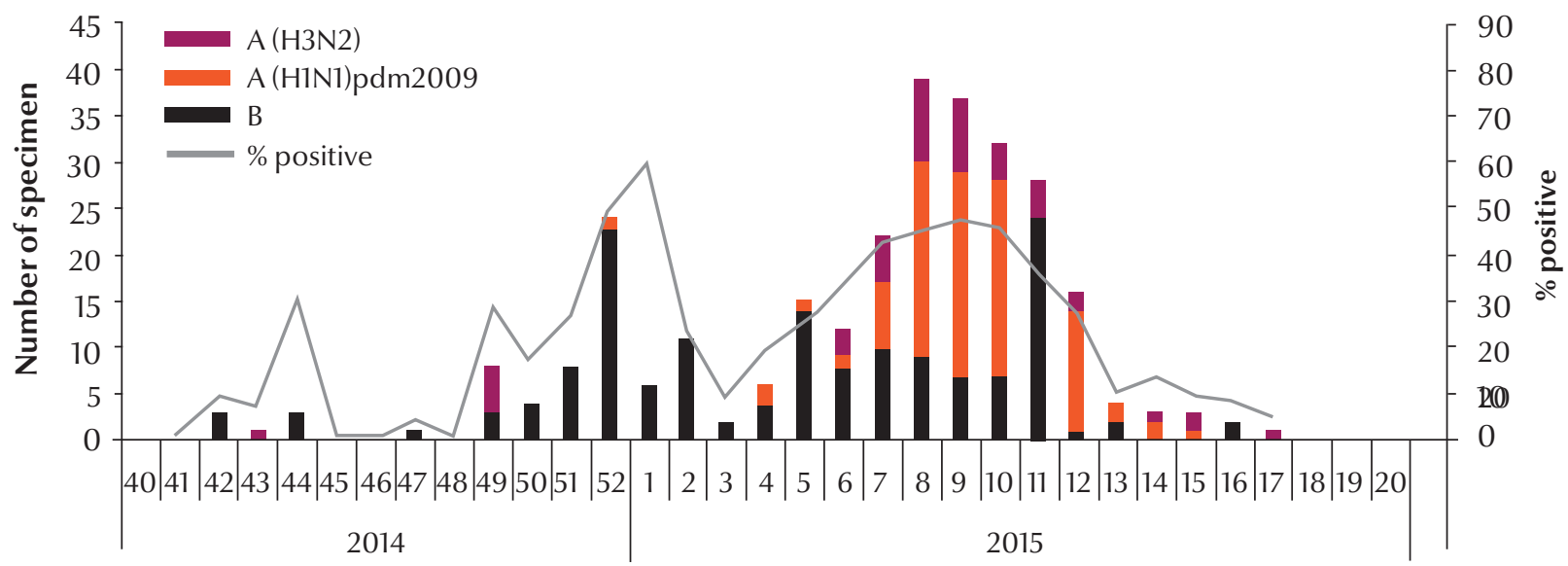

Week and year

Figure 1 Estimated seasonal pattern of laboratory-confirmed influenza activity in Tunisia in seasons 2012-13, 2013-14 and 2014-15 according to virus subtype

than among persons in the other 2 age groups examined.

The estimated seasonal pattern of laboratory-confirmed influenza activity for the 3 seasons are shown in Figure 2. During the most recent season (2014-2015), 1038 samples were tested, of which 291 were positive for influenza viruses (positivity rate $28.0 \%)$. The seasonal distribution was: $A(\mathrm{H} 3 \mathrm{~N} 2) 15.5 \%$; $\mathrm{A}(\mathrm{H} 1 \mathrm{~N} 1)$ pdm2009 39.2\%; and B virus 45.3\%. In comparison, in the 2012-2013 season, 924 samples were collected, positivity rate $37.4 \%$, and the seasonal distribution of virus subtypes was: $\mathrm{A}(\mathrm{H} 3 \mathrm{~N} 2)$ 6.1\%; A (H1N1)pdm2009 50.1\%; and B 38.3\%; and in the 2013-2014 season, 


\begin{tabular}{|c|c|c|c|}
\hline Age (years) & $\begin{array}{c}2012 / 13 \\
\%(95 \% \mathrm{Cl})\end{array}$ & $\begin{array}{c}2013 / 14 \\
\%(95 \% \mathrm{Cl})\end{array}$ & $\begin{array}{c}2014 / 15 \\
\%(95 \% \mathrm{Cl})\end{array}$ \\
\hline & $P<0.0001$ & $P<0.0001$ & $P<0.0001$ \\
\hline $0-5$ & $2.77(2.71-2.82)$ & $0.88(0.85-0.91)$ & $1.91(1.87-1.96)$ \\
\hline $6-16$ & $4.50(4.43-4.58)$ & $1.29(1.25-1.33)$ & $2.80(2.75-2.86)$ \\
\hline$\geq 16$ & $2.94(2.91-2.97)$ & $0.76(0.75-0.78)$ & $1.81(1.79-1.84)$ \\
\hline Total & $3.16(3.13-3.18)$ & $0.86(0.85-0.87)$ & $1.98(1.97-2.00)$ \\
\hline
\end{tabular}

514 samples were collected, positivity rate $12.1 \%$, and the seasonal distribution of virus subtypes was: $\mathrm{A}(\mathrm{H} 3 \mathrm{~N} 2)$ 96.8\%; $\mathrm{A}(\mathrm{H} 1 \mathrm{~N} 1) p d m 2009$ 1.6\%; and B $1.6 \%$.

The basic reproduction numbers (R0) were estimated from the analytical solution of the model and data derived from surveillance. They were 1.18, 1.04 and 1.11 for 2012-2013, 2013-2014 and 2014-2015 respectively (Table 3). The computed symptomatic attack rates (Table 4) were very close to the estimated rates used in the computation of R0; this can be interpreted as a sort of validation of the modelling process. From the estimated numbers of symptomatic cases, the attack rates and the R0 values for the 2012-2015 seasons, we estimated that the critical proportion of the population that should be vaccinated was $15 \%$ for the $2012-2013$ season, 4\% for the 2013-2014 season and 10\% for the 2014-2015 season. The critical vaccination proportion needed to stop the influenza epidemic was calculated as described in equation 17.

\begin{tabular}{|c|c|c|c|}
\hline \multirow[t]{2}{*}{ Item } & \multicolumn{3}{|c|}{ Influenza season } \\
\hline & $2012 / 13$ & 2013/14 & 2014/15 \\
\hline Population size & 10886500 & 10982754 & 11151874 \\
\hline $\begin{array}{l}\text { Estimated no. } \\
\text { symptomatic } \\
\text { cases }\end{array}$ & 2057657 & 608009 & 1384115 \\
\hline $\begin{array}{l}\text { Symptomatic } \\
\text { attack rate }\end{array}$ & 0.19 & 0.06 & 0.12 \\
\hline$S_{\infty}$ & 7800015 & 10070741 & 9075702 \\
\hline $\mathrm{R}_{0}$ & 1.18 & 1.04 & 1.11 \\
\hline
\end{tabular}

significantly higher than observed from data reported by the National Influenza Centre. These findings might indicate that the mortality rates associated with influenza are underestimated in our surveillance system. The nomination of 6 SARI surveillance sites representing the whole country is expected to improve the validity of these estimations. Moreaccurate and representative morbidity and mortality data will serve to further refine our model.

The derivation of the critical fraction of the population to be vaccinated is a key public health parameter that is useful in indicating the extent of the control challenge of infectious diseases. Although not very accurate because of the difficulty in controlling all sources of heterogeneity, it can be extremely helpful in guiding policy-makers on the importance of needs. In our study, the critical fraction of the total population that should be vaccinated against influenza ranged from $4 \%$ to $15 \%$, which is significantly higher than the observed coverage in Tunisia (about 3\% according to the estimates of the National Programme of Control for Seasonal Influenza in Tunisia), particularly during high transmission seasons. This fraction assumes 100\% efficacy of protection against infection and a homogeneous transmission risk. These assumptions are, unfortunately, over-simplistic, and more epidemiological studies are required to better assess high-risk groups and derive realistic protection rates. These critical fractions would be higher among high-risk groups. Serological studies of immune protection in the population and genetic comparisons of vaccine strains with the circulating strains are required to evaluate the extent of effective protection during a given season. This indicates the importance of strengthening the biological capacity of national influenza centres and the rapid integration of further information into real risk assessments. Studies of risk factors related to severe clinical expression of influenza infection 


\begin{tabular}{lccccccc}
\hline \multicolumn{2}{l}{$\begin{array}{l}\text { Table } 4 \text { Mortality and morbidity indicators as outcomes of the model simulations, Tunisia 2012-2015 influenza seasons } \\
\text { Season }\end{array}$} & R0 & $\begin{array}{c}\text { Susceptible } \\
\text { population }\end{array}$ & $\begin{array}{c}\text { Infected } \\
\text { cases (No.) }\end{array}$ & $\begin{array}{c}\text { Symptomatic } \\
\text { clinical cases } \\
\text { (No.) }\end{array}$ & $\begin{array}{c}\text { Deaths (No.) } \\
\text { Clinical attack } \\
\text { rate }\end{array}$ & $\begin{array}{c}\text { Symptomatic } \\
\text { clinical attack } \\
\text { rate }\end{array}$ \\
$2012 / 13$ & 1.18 & 7824000 & 3176100 & 2117340 & 212 & 0.29 & 0.19 \\
$2013 / 14$ & 1.04 & 10164600 & 835374 & 556920 & 56 & 0.08 & 0.05 \\
$2014 / 15$ & 1.11 & 8894240 & 2105770 & 1403850 & 140 & 0.19 & 0.13 \\
\hline
\end{tabular}

A simulation was done for each of the 3 seasons; values were taken at equilibrium.

would also better identify the high-risk groups, such as elderly patients with comorbidities and pregnant women, for a targeted vaccination strategy. Inclusion of the private sector in the surveillance of influenza is highly recommended to improve the representativeness of surveillance estimations.

More-realistic versions of this model and improved estimates of parameters from epidemiological surveillance data and surveys will strengthen the strategy of using mathematical models to estimate the real burden of influenza and to predict the impact of vaccination programmes and treatment strategies on the future dynamics of influenza infection, disease and mortality (11-13). Future versions of the model might consider heterogeneities among high-risk groups (infants versus elderly or pregnant women for example), and simulation of different vaccination strategies (e.g. integration of influenza vaccine into the Expanded Programme on Immunization versus prioritizing the elderly or pregnant women).

Our study demonstrated the relevance of mathematical modelling for the estimation of influenza burden in Tunisia; further refinement is needed to improve our estimations and better orient public health interventions for better control. The quality of surveillance data is key to ensuring the validity and reproducibility of the model.

\section{Influenza Surveillance Group in Tunisia:}

R.T. Dellagi, M. Zorraga, N. Abdeddaiem, T. Atawa, S.M. El Bekri, Primary Health Care Directorate, Ministry of Public Health of Tunisia, Tunis, Tunisia. J. Ben Khelil, M. Besbes, Department of Intensive Care Medicine, Abderrahmen Mami Hospital, Ariana, Tunisia. M.A. Ben Hadj Kacem, Virology Unit, Microbiology LAboratory, National Influenza Centre, Charles Nicolle's Hospital, Tunis Tunisia. K. Mnif, N. Ben Jaballah, Paediatric
Intensive Care Unit, Children's Hospital of Tunis, Tunisia. S. Aissa, Pulmonology Department Diseases, Farhat Hached Hospital, Sousse, Tunisia. M. Boussarsar, Department of Intensive Care Medicine, Farhat Hached Hospital, Sousse, Tunisia. M. Ben Jemaa, Department of Infectious Diseases, Hedi Chaker University Hospital of Sfax, Sfax University, Tunisia. M. Bouaziz, Unit of Medical Resuscitation, Habib Bourguiba University Hospital, Sfax, Tunisia.

\section{Acknowledgment}

Funding: This study was funded by the WHO Regional Office for the Eastern Mediterranean (Project registration: 2015/542761-0; Unit Reference PED/ DCD) and the Centres for Disease Control and Prevention, Atlanta, United States of America (Grant Number 1U51IP000822, 2013-2018).

Competing interests: None declared.

\section{References}

1. El Moussi A, Pozo F, Ben Hadj Kacem MA, Ledesma J, Cuevas MT, Casas I et al. Virological surveillance of influenza viruses during the 2008-09, 2009-10 and 2010-11 seasons in Tunisia. PLoS One. 2013;8(9):e74064.1

2. Global epidemiological surveillance standards for influenza. Geneva: World Health Organization; 2013 (http://www.who. int/influenza/resources/documents/WHO_Epidemiological_Influenza_Surveillance_Standards_2014.pdf, accessed 7 September 2016).

3. A manual for estimating disease burden associated with seasonal influenza. Geneva: World Health Organization; 2015 (http://apps.who.int/iris/bitstre am/10665/178801/1/9789241549301_eng.pdf?ua=1\&ua=1, accessed 7 September 2016).

4. Recensement Général de la Population et de l'Habitat 2014: principaux indicateurs. République Tunisienne, Institut National de Statistiques; 2015 (http://www.ins.tn/sites/default/ files/publication/pdf/rgph-chiffres-web_0.pdf, accessed 27 June 2016).

5. Brauer F, Van den Driessche P, Wu J. Mathematical epidemiology. Berlin: Springer; 2008.

6. Brauer F. Some simple epidemic models. Math Biosci Eng. 2006;3(1):1-15.

7. Longini IM, Halloran ME, Nizam A, Yang Y. Containing pandemic influenza with antiviral agents. Am J Epidemiol. 2004;159(7):623-33.

8. van den Driessche P, Watmough J. Reproduction numbers and subthreshold endemic equilibria for compartmental models of disease transmission. Math Biosci. 2002;180:29-48.

9. Arino J, Brauer F, van den Driessche P, Watmough J, Wu J. Simple models for containment of a pandemic. J R Soc Interface. 2006;3(8):453-7. 
10. Arino J, Brauer F, van den Driessche P, Watmough J, Wu J. A model for influenza with vaccination and antiviral treatment. J Theor Biol. 2008;253(1):118-30.

11. Balicer RD, Huerta M, Davidovitch N, Grotto I. Cost-benefit of stockpiling drugs for influenza pandemic. Emerg Infect Dis. 2005;11:1280-2.
12. Ferguson NM, Cummings DAT, Cauchemez S, Fraser C, Riley S, Meeyai A, et al. Strategies for containing an emerging influenza pandemic in Southeast Asia. Nature. 2005;437:209-14.

13. Gani R, Hughes H, Griffin T, Medlock J, Leach S. Potential impact of antiviral use on hospitalizations during influenza pandemic. Emerg Infect Dis. 2005;11:1355-62. 